\title{
Internal transport barrier driven by redistribution of energetic ions
}

\author{
K.L. Wong ${ }^{1}$, W.W. Heidbrink ${ }^{2}$, E. Ruskov ${ }^{2}$, C.C. Petty ${ }^{3}$, \\ C.M. Greenfield ${ }^{3}$, R. Nazikian ${ }^{1}$ and R. Budny ${ }^{1}$ \\ ${ }^{1}$ Plasma Physics Laboratory, Princeton University, Princeton, NJ 08543, USA \\ 2 Department of Physics, University of California, Irvine, CA 92697, USA \\ ${ }^{3}$ General Atomics, PO Box 85608, San Diego, CA 92186, USA
}

Received 27 May 2004, accepted for publication 21 October 2004

Published 31 December 2004

Online at stacks.iop.org/NF/45/30

\begin{abstract}
Alfvén instabilities excited by energetic ions are used as a means to reduce the central magnetic shear in a tokamak via redistribution of energetic ions. When the central magnetic shear is low enough, ballooning modes become stable for any plasma pressure gradient and an internal transport barrier (ITB) with a steep pressure gradient can exist. This mechanism can sustain a steady-state ITB as demonstrated by experimental data from the DIII-D tokamak. It can also produce a shear in toroidal and poloidal plasma rotation. Possible application of this technique to use the energetic $\alpha$ particles for improvement of burning plasma performance is discussed.
\end{abstract}

PACS numbers: 52.55.Pi, 52.35.Bj, 52.35.Py

\section{Introduction}

After ignition, a DT fusion reactor is supposed to be sustained by $\alpha$ particle heating, in which the $\alpha$ particle energy is transferred to the thermal electrons and ions via Coulomb collisions. Because of their high birth energy $(3.5 \mathrm{MeV})$, most of the $\alpha$ particle energy goes to the electrons, which is undesirable because electrons do not fuse and they have poor confinement properties in existing tokamaks. The concept of ' $\alpha$ channelling' was proposed more than a decade ago to channel the $\alpha$ power to accomplish more useful functions, e.g. current drive or ion heating, instead of electron heating. Poloidally and toroidally propagating lower hybrid waves were first proposed to extract the $\alpha$ particle energy [1] and a twowave scheme, a combination of ion Bernstein wave (IBW) and toroidal Alfvén eigenmode (TAE) [2], was proposed later. Diffusion paths were identified to convert energetic $\alpha$ particles in the core to low energy $\alpha$ particles at the edge. The fusion power density can double if $75 \%$ of the $\alpha$ particle energy is channelled to the $\mathrm{D} / \mathrm{T}$ ions [3]. However, some wave characteristics are quite demanding, and they have not yet been realized in laboratory experiment. Although current drive was discussed in the previous work [1], so far, the focus has been placed on $\mathrm{D} / \mathrm{T}$ ion heating $[2,3]$. In this paper, we explore another possibility and show that TAE or other Alfvén modes excited by the $\alpha$ particle pressure gradient can reduce or reverse the central magnetic shear and drive a plasma flow shear for better plasma confinement. If one can use the energetic $\alpha$ particles to shape the $q$-profile and raise the plasma $\beta$ limit by $40 \%$ in a reactor, the fusion power density can double. This accomplishment rivals complete channelling of $\alpha$ particle energy to ion heating $[2,3]$. Experimental data from DIII-D are presented to demonstrate the feasibility of this scheme.

Negative central magnetic shear (NCS) is highly desirable for tokamak confinement because it eliminates ballooning and interchange magnetohydrodynamic (MHD) instabilities and large pressure gradients can exist in the plasma core without turbulent plasma transport. Internal transport barriers (ITB) are routinely produced in NCS plasmas and ion channel transport can be reduced to the neoclassical level $[4,5]$. This is attributed to the poloidal velocity shear or the gradient of the radial electric field $\left(E_{\mathrm{r}}\right)$ as well as to magnetic well stabilization which are large enough to stabilize the ion temperature gradient (ITG) turbulence [6-8]. Although these experiments are usually performed in NCS plasmas, they also work in plasmas with flat or slightly positive magnetic shear [9] represented by the shaded region of the $s-\alpha$ diagram depicted in figure 1 because of the magnetic well stabilization effect [10].

NCS plasmas in tokamaks are usually produced by injecting neutral beams early in the shot to raise the electron temperature during the current ramp up phase when the current density profile is hollow. The high electron temperature will slow down the current penetration process, so that one can have a NCS configuration over an extended period of time, but eventually (seconds later for existing tokamaks), it will evolve into a plasma with normal positive magnetic shear. When 


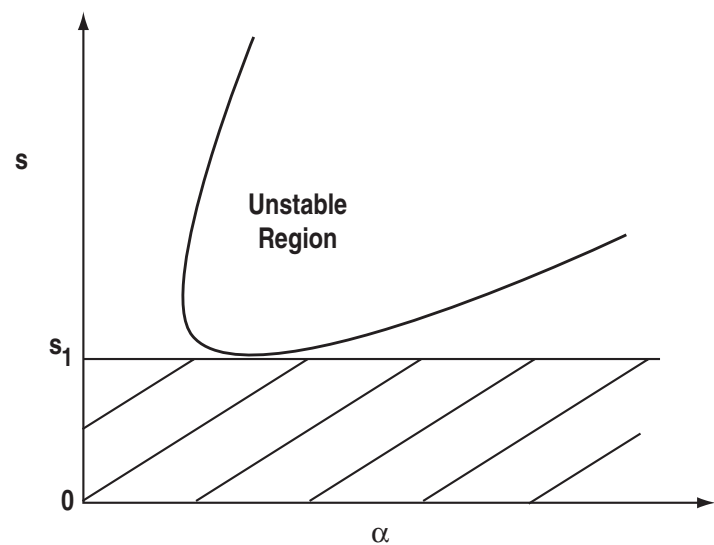

Figure 1. Stability diagram for high- $n$ ballooning modes; $s$ denotes magnetic shear, and $\alpha$ denotes the plasma pressure gradient. Ballooning modes are stable for all values of $\alpha$ when $s<s_{1}$ (shaded region).

$q_{\text {min }}$ drops below some low order rational number $m / n$, MHD activity at $q=m / n$ appears. Finally, $q(0)$ gets below 1 , and sawtooth activity dominates the plasma core. This is the typical time history of a transient ITB in existing tokamak experiments where the $q$-profile continuously evolves towards $q(0) \sim 1$. In order to sustain a steady-state ITB with $q_{\min }>1$, some offaxis current drive scheme is necessary to arrest the evolution of the $q$-profile. Data from DIII-D show that redistribution of energetic ions from Alfvén instabilities can be such a scheme.

Neutral beams injected into low-density plasmas can produce a high population of energetic ions because of the low electron drag, and Alfvén instabilities can be excited which eject the energetic ions from the plasma core to the periphery [11-13]. When the beam deposition profile is centrally peaked, the unstable Alfvén modes always propagate toroidally parallel to the plasma current. This is because the modes have very long parallel wavelengths $\left(k_{\|} / k_{\theta}<B_{\theta} / B_{\phi}\right)$, and they are driven by the fast ion pressure gradient so that they must propagate poloidally in the ion diamagnetic drift direction. Geometry dictates the direction of $k_{\phi}$ as explained in [13]. Therefore, only co-moving (toroidal velocity parallel to the plasma current) fast ions can resonate with the excited Alfvén modes and get ejected from the core to the edge. This happens within a small fraction of a millisecond. These ejected co-moving fast ions are not sensitive to ITG turbulence because of their large orbits; they can stay in the outer region of the plasma, drive a cocurrent that lowers the $q$-value there. These fast ions come from the core; losing them results in a reduced non-inductive current in the core and would raise the $q$-value there. This happens in the current diffusion time scale (about $1 \mathrm{~s}$ in existing tokamaks). Therefore, the redistribution of these energetic ions due to the Alfvén modes provides a mechanism for off-axis current drive for the reduction or reversal of the magnetic shear in the plasma core. This process is schematically illustrated in figure 2. In section 2, we present experimental data from the DIII-D tokamak showing the existence of this process. Section 3 investigates the possibility of deploying this scheme in a tokamak fusion reactor. More general discussions on
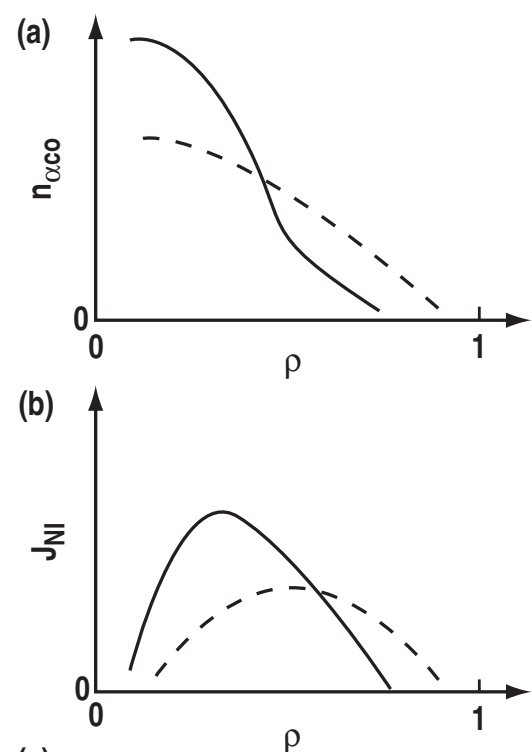

(c)

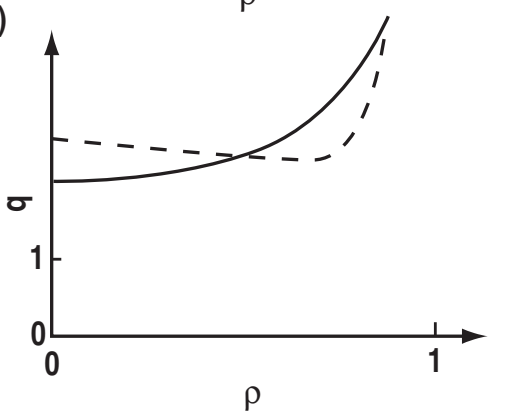

Figure 2. Schematic diagrams to illustrate formation of NCS configuration due to redistribution of co-moving fast ions. Solid (- - - ) line denotes the profile before (after) redistribution.

(a) Density profile of co-moving fast ions. (b) Non-inductive current density profile. (c) $q$ profile.

improvements of this scheme are given in section 4 and a summary is given in section 5 .

\section{Experimental data from DIII-D}

Experimental evidence of the above process was found in the DIII-D tokamak. When one co-beam source ('left' source with $1.15 \mathrm{~m}$ tangency radius) was injected into a $700 \mathrm{kA}$ low-density plasma with $n_{\mathrm{e}}<10^{19} \mathrm{~m}^{-3}, B=1.8 \mathrm{~T}$ (shot 92755), some high-frequency MHD activities appear in the Mirnov coil signal as depicted in figure 3. These are Alfvén eigenmodes $(\mathrm{AE})$ at $60 \mathrm{kHz}(n=1)$ and $85 \mathrm{kHz}(n=2)$ excited by the beam ions. Both modes propagate parallel to the plasma current. These modes appear only during neutral beam injection into low-density target plasmas. Their appearance strongly correlates with high beam beta. They are more readily excited by the 'left' beams than the 'right' beams. (The injection velocities of the beam ions from the 'left' beams have a larger component parallel to the magnetic field than those from the 'right' beams.) The typical beam ion parallel velocity is about $0.4 \mathrm{~V}_{\mathrm{A}}$. The instabilities shown in figure 3 appear in bursts that typically persist for about $1 \mathrm{~ms}$. After $2200 \mathrm{~ms}$, the $n=1$ bursts occur every few 

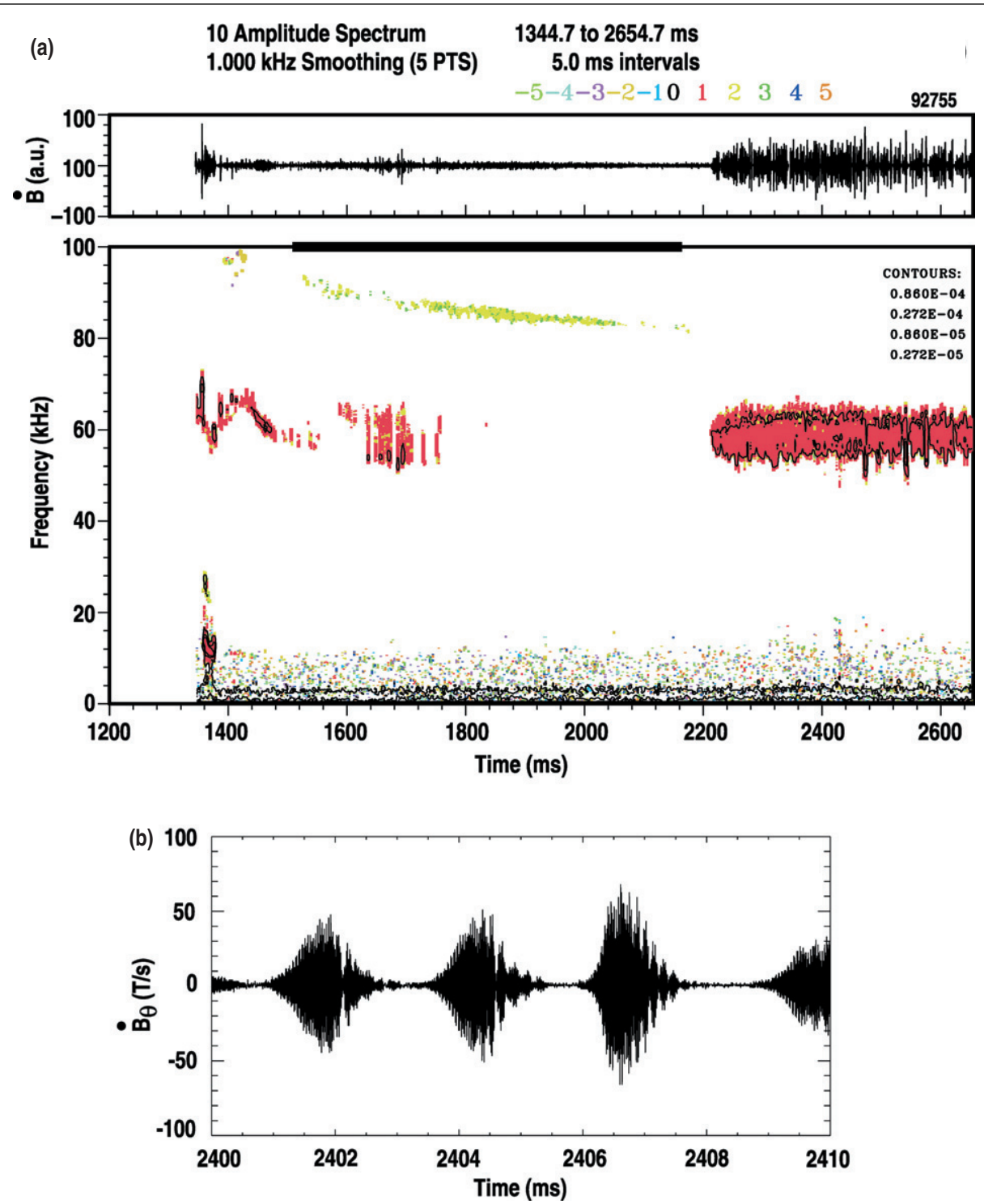

Figure 3. (a) Mirnov coil signal from pulse 92755 showing the $n=1(\sim 60 \mathrm{kHz})$ and the $n=2(\sim 85 \mathrm{kHz})$ modes propagating parallel to the plasma current. (b) Mirnov coil signal in expanded time scale showing the bursting nature of the mode.

milliseconds. With each burst, the frequency sweeps down about $10 \mathrm{kHz}$ in approximately $0.5 \mathrm{~ms}$. The mode frequency of about $60 \mathrm{kHz}$ is much higher than the central toroidal rotation frequency of about $20 \mathrm{kHz}$, so these instabilities propagate in the plasma frame. The frequency is significantly lower than the nominal TAE frequency of $200 \mathrm{kHz}$, so these are probably not TAEs. Modes with rapid frequency chirping are relatively rare in DIII-D but similar instabilities were previously reported [14]. They are probably some type of energetic particle modes (EPM) [15]. Empirically in DIII-D, low plasma density and weak magnetic shear are conducive to beam-driven instabilities $[16,17]$. For those modes localized in the plasma core, their mode amplitude can be too small at the plasma edge for detection by Mirnov coils. Since the discovery of TAE modes $[11,12]$, the Alfvén spectrum has become much more complicated when more physical mechanisms are incorporated. Many modes are possible in this frequency range. Definitive mode identification requires high quality data on the spatial mode structure and detailed knowledge of the fast ion distribution function which are absent in most of the reported experiments including the one presented here. The $n=2$ mode near half the TAE frequency would have been called the BAE mode 10 years ago. The real nature of BAE was carefully investigated recently $[16,17]$, where the complexity of mode identification is described. There is no need to get into these details again for this experiment partly because there are not enough data for definitive mode identification. Moreover, all these Alfvén modes excited by the centrally peaked fast ions have the same effect, namely, they flatten the fast ion pressure profile [18] and reduce or reverse the central magnetic shear. The mechanism proposed here does not depend on any specific AE mode; it works for all the modes driven by a centrally peaked fast ion pressure profile.

An ITB is formed in shot 92755 at $\rho \sim 0.4$ with $T_{\mathrm{i}}(0)>10 \mathrm{keV}, T_{\mathrm{e}}(0)>4 \mathrm{keV}$. The $q$-profile in the core remains flat throughout the beam pulse; $q$ at the core drops very slowly with $q(0)>1.8$ until the neutral beam is turned off at $2.8 \mathrm{~s}$ (figure $4(a)$ ). This is distinctly different from 

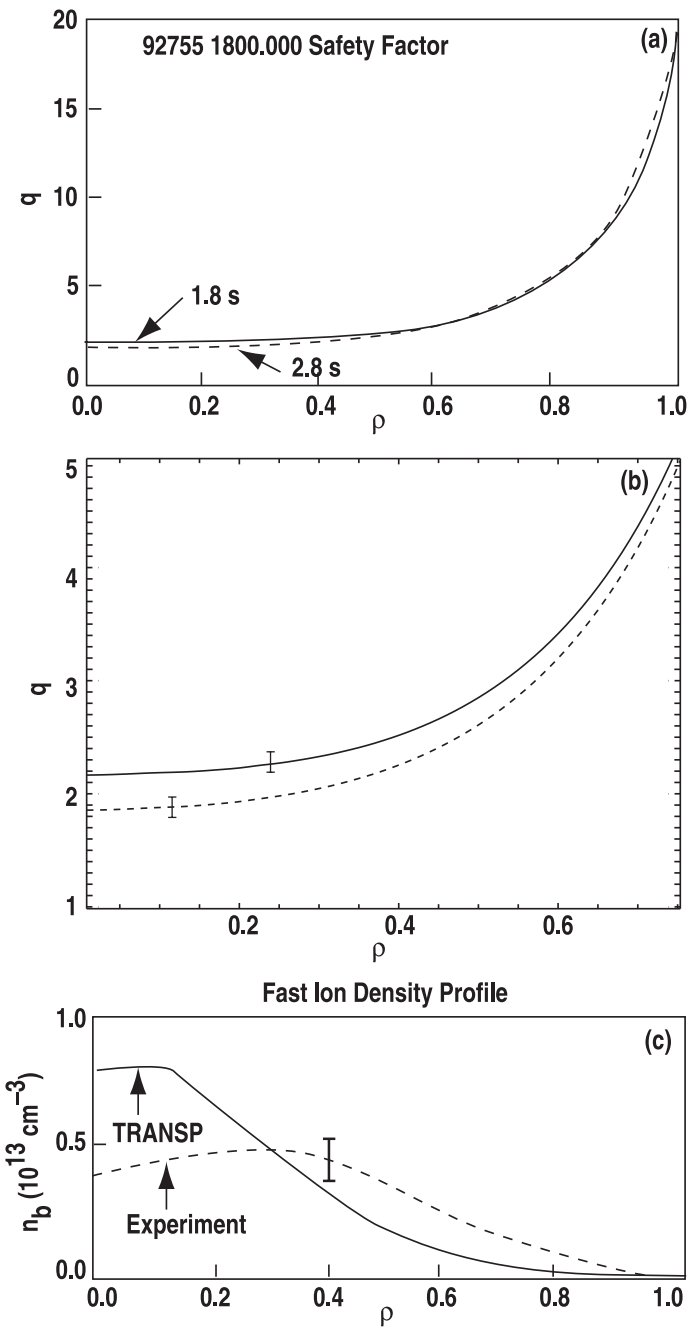

Figure 4. (a) $q$-profile for shot 92755 at 1.8 and $2.8 \mathrm{~s}$. (b) $q$-profile in more sensitive scale with error bars representing the experimental uncertainty of \pm 0.1 in the $q$ values measured by MSE.

(c) Calculated beam ion density profile compared to that obtained from the experimental data.

those shots without AEs where $q(0)$ drops to 1 very quickly. Comparison between similar pulses indicates that the AEs are responsible for this behaviour. This is further illustrated in figure 7 which we will relay later. The calculated beam deposition profile is centrally peaked. The 'experimental' beam ion density profile in figure $4(c)$ is obtained from the difference between the thermal plasma pressure profile from plasma kinetic measurements and the total pressure profile from equilibrium reconstruction by the EFIT code with motional Stark effect (MSE) data. It is significantly broader than the modelling results from TRANSP [19] and ONETWO [20] because of the redistribution of beam ions by the AEs which is not incorporated into the modelling. A typical value used for the beam ion diffusivity in this modelling is $D_{\mathrm{b}} \sim 1 \mathrm{~m}^{2} \mathrm{~s}^{-1}$, which is substantially less than what is needed to account for the large loss rate due to the AEs (compare with the values depicted in figure 6(a)). The non-inductive current due to the beam ions are also broader, which explains the flat $q(r)$ in the plasma core. We see such a discrepancy only when AEs are present. In pulses without (a)
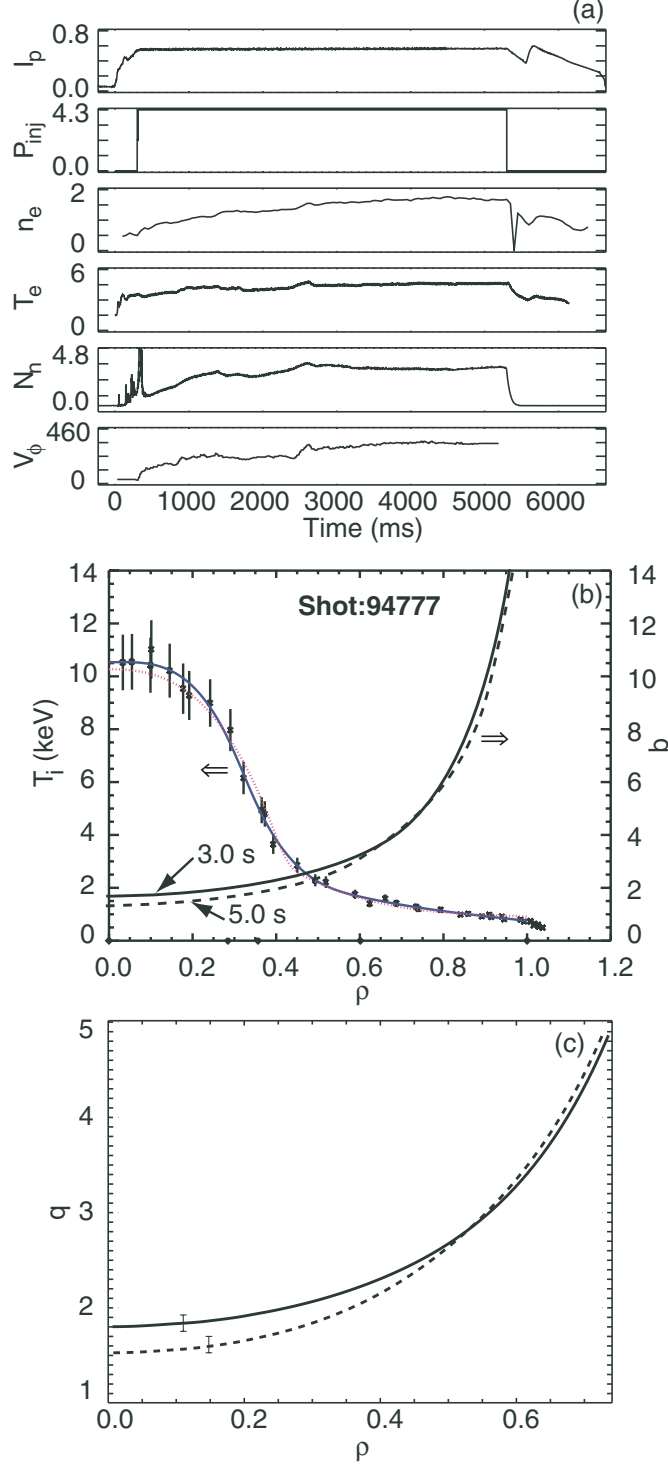

Figure 5. Plasma characteristics for shot 94777. (a) Waveforms for plasma current $I_{\mathrm{p}}(\mathrm{MA})$, injected neutral beam power $P_{\text {inj }}(\mathrm{MW})$, line-averaged electron density $n_{\mathrm{e}}\left(10^{19} \mathrm{~m}^{-3}\right)$, core electron temperature $T_{\mathrm{e}}(\mathrm{keV})$, total neutron emission rate $N_{\mathrm{n}}$ $\left(10^{14}\right.$ neutrons $\left.\mathrm{s}^{-1}\right)$ and core toroidal rotation velocity $v_{\phi}\left(\mathrm{km} \mathrm{s}^{-1}\right)$. (b) Ion temperature profile and $q$-profile at $t=3.0$ and $5.0 \mathrm{~s}$. (c) $q$-profile in more sensitive scale with error bars representing the experimental uncertainty of \pm 0.1 in the $q$ values measured by MSE.

AEs, the experimental and modelled beam ion densities are in agreement.

High-performance plasma with a quasi steady-state ITB was produced in DIII-D (shot 94777) by two co-beam sources injected into a $600 \mathrm{kA}$ plasma with $n_{\mathrm{e}} \sim 1.5 \times 10^{19} \mathrm{~m}^{-3}$, $B=1.9 \mathrm{~T}, q(0)>1.6$. Only the 'left' beam sources were used. These sources have the same tangency radii, of $1.15 \mathrm{~m}$, so that the same beam voltage and beam power from different sources would deliver the same torque to the plasma. The central magnetic shear stays flat until the beam is turned off. The ITB at $\rho \sim 0.4$ lasts until the end of the beam pulse. This is similar to the previous case shown in figure 4 except that the beam pulse is $5 \mathrm{~s}$ long instead of $2 \mathrm{~s}$. Figure $5(a)$ shows 

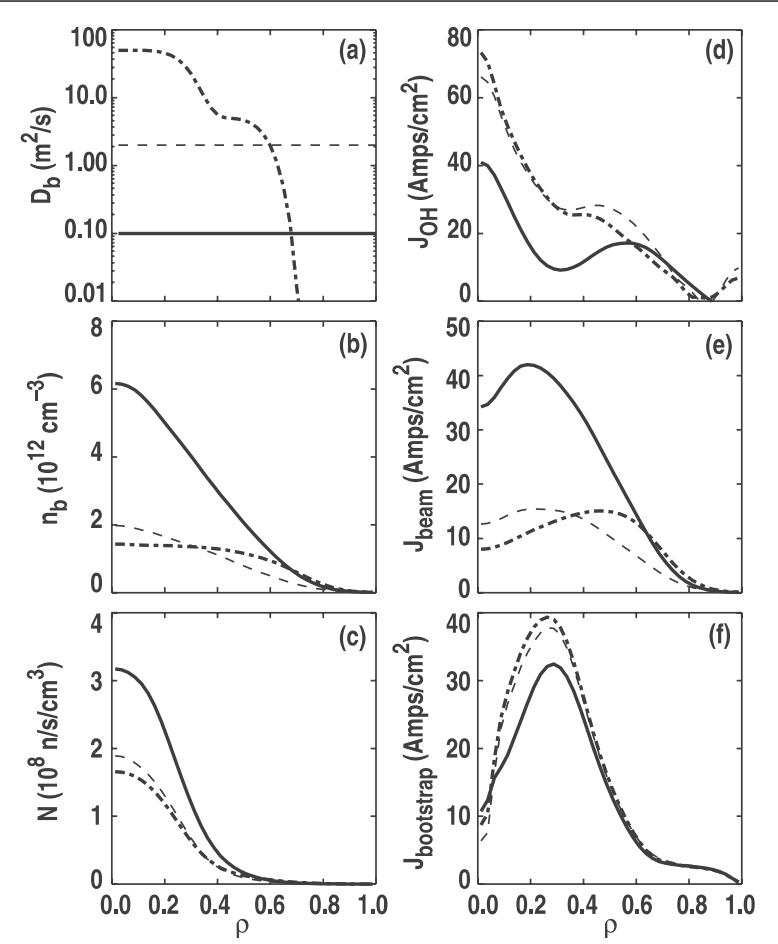

Figure 6. TRANSP modelling results for pulse 94777 with three different values of diffusion coefficient $D_{\mathrm{b}}$ for the beam ions. (a) Radial profiles of $D_{\mathrm{b}}$. (b) Corresponding profiles of beam ion density $n_{\mathrm{b}}$ calculated from the three different fast ion diffusion coefficients. (c) Neutron emissivity profiles $N$. Integration over the plasma volume yields the total neutron rate. $(d)$ Ohmic current density profiles $J_{\mathrm{OH}}$. (e) Beam-driven current density profiles $J_{\text {beam }}$. $(f)$ Bootstrap current density profiles $J_{\text {bootstrap }}$.

the waveforms for the plasma current, neutral beam power, electron density, electron temperature, neutron rate and the toroidal rotation velocity. As depicted in figure 5, the electron and ion temperatures are almost independent of time from 3 to $5 \mathrm{~s}$. However, this is not exactly a steady-state plasma; $q(0)$ drops very slowly in time, and $n_{\mathrm{e}}$ goes up monotonically inside the ITB which is typical for these plasmas with beam fuelling. This causes the slow broadening of the beam deposition profile as well as the toroidal rotation velocity profile at later time. The neutron rate in this shot is much lower than one would expect from the beam power and the target density because the AEs eject the beam ions from the plasma core. This process is modelled by a large beam ion diffusivity $D_{\mathrm{b}}$; a very large anomalous diffusion coefficient for the fast ions is needed $\left(D_{\mathrm{b}} \sim 50 \mathrm{~m}^{2} \mathrm{~s}^{-1}\right)$ in order to match the observed neutron rate. A better match with experimental data is achieved by allowing $D_{\mathrm{b}}$ to have some radial dependence. Since fast ions are ejected from the plasma core, we choose a form of $D_{\mathrm{b}}$ that peaks in the plasma core. Figure 6 shows the beam ion density, neutron emissivity and various components of the current density calculated from the TRANSP modelling code with the three different $D_{\mathrm{b}}$ profiles depicted in figure 6(a) subject to the tight constraints from all the experimental measurements. The magnetic shear in the plasma core of pulse 94777 is almost zero but not negative; the presence of the ITB indicates that the shear is low enough to avoid ballooning modes.

The major effect of the AEs is the expulsion of co-moving energetic ions from the plasma core which reduces the current

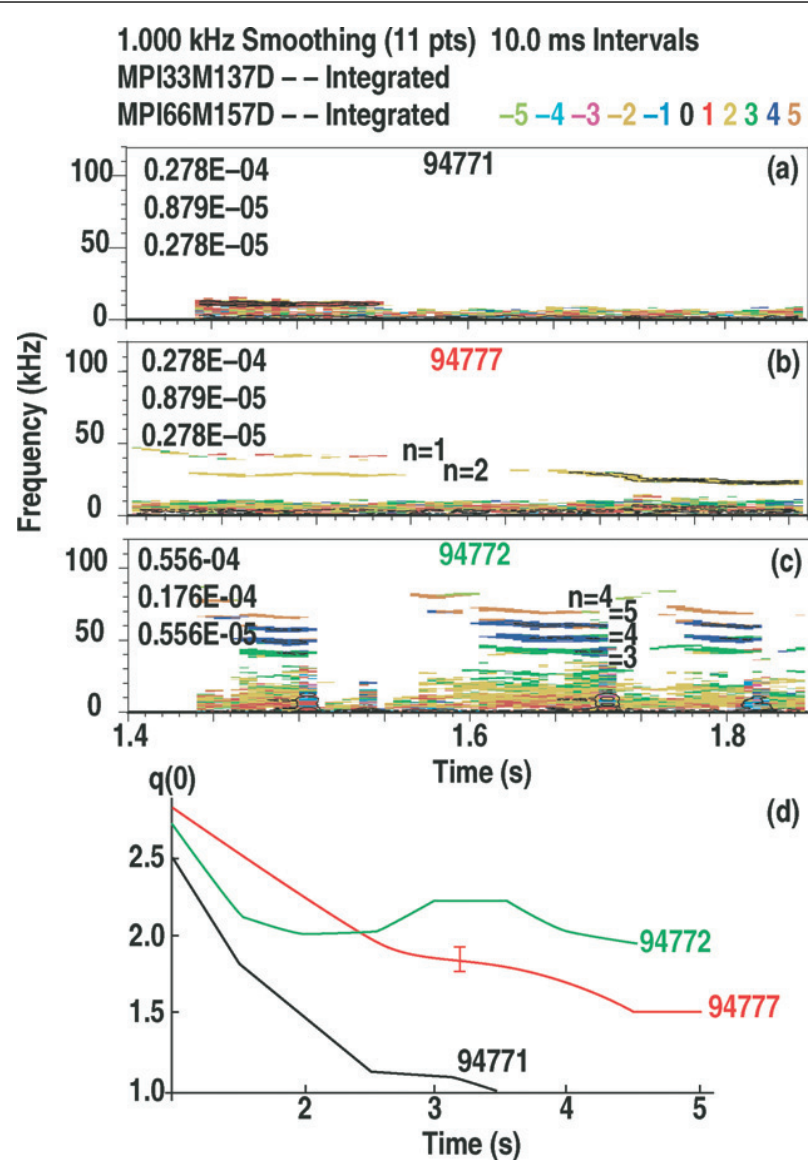

Figure 7. Correlation between AE activities and the evolution of $q(0)$. Frequency spectrum of the Mirnov coil signal for $(a)$ pulse 94771, which has a $3.1 \mathrm{MW}$ deuterium neutral beam at $70.4 \mathrm{kV}$ and the AEs are marginally stable, $(b)$ pulse 94777 , which has a $4.3 \mathrm{MW}$ deuterium neutral beam at $73.5 \mathrm{kV}$ and two Alfvén modes that are unstable, $(c)$ pulse 94772 , which has a 4.35 MW deuterium neutral beam at $73.5 \mathrm{kV}$ and a startup density that is slightly higher than in pulse 94777 . (d) Evolution of $q(0)$ for the above three pulses.

density in the core. If these fast ions stay in the outer region of the tokamak, they will increase the current density which reduces the $q$ value there. However, this effect is not so obvious owing to the larger plasma volume and the loss of those particles with large orbits. Since $q(0)=\left(2 B_{\phi} / \mu_{\mathrm{o}} R\right) / J(0)$, the best way to detect the proposed mechanism depicted in figure 2 is to correlate the value of $q$ in the vicinity of the magnetic axis with the AE activities excited by the fast ions. Figure 7 compares three similar plasma pulses with different levels of AE activities. These plasmas are produced in the same shot sequence with the same plasma current, toroidal magnetic field, major radius and plasma shape. AEs are marginally stable in shot 94771 , and $q(0)$ reaches 1.0 at $t=3.4 \mathrm{~s}$. AEs are unstable in shot 94777 where the injected beam power is $25 \%$ higher than in shot 94771 and $q(0)$ stays above 1.6 until the end of the $5 \mathrm{~s}$ long beam pulse. At the same beam power as in shot 94777 but slightly higher startup density, AE activities are even stronger in shot $94772\left(\beta \sim 1 \%, \beta_{\mathrm{N}} \sim 1.8\right)$ and $q(0)$ stays above 2.0 through out the $5 \mathrm{~s}$ long beam pulse. The mode numbers shown in figure $7(c)$ are determined from data at $1.65 \mathrm{~s}$. At $t=1.58 \mathrm{~s}$, clusters of modes at $70 \mathrm{kHz}$ $(n=4), 81 \mathrm{kHz}(n=5)$ and $91 \mathrm{kHz}(n=6)$ appear. Their frequency in the plasma frame is $31 \mathrm{kHz}$, which is definitely 
propagating, but much lower than the nominal TAE frequency of about $120 \mathrm{kHz}$. The frequency is near the resonant KBM frequency described in Gorelenkov and Heidbrink [17]; these are probably EPM modes where the energetic particles play an important role. As mentioned earlier, detailed classification of these modes is not necessary here because they all tend to flatten the energetic ion pressure gradient. The behaviour of $q(0)$ depicted in figure 7 is a strong indication that the mechanism shown in figure 2 is at work. The toroidal mode numbers of the AEs in all these pulses are measured by a toroidal array of magnetic probes. They all propagate parallel to the plasma current as expected.

In order to investigate this in a more quantitative manner, we analyse pulses 94771 and 94777 with the TRANSP code and compare them in detail (pulse 94772 is not chosen for detailed TRANSP analysis primarily because some of its Thomson scattering data files are missing). The AE activities in the experiment are mainly controlled by the neutral beam power. The injected neutral beam power in shot 94771 is $25 \%$ lower than that in shot 94777 so that the AEs are marginally stable. Because of the different heating power, it is inevitable that there are differences in the plasma parameters. A perfect way to make a quantitative evaluation of the $\mathrm{AE}$ effect is to make the AEs come and go and keep everything else exactly the same. Unfortunately, this cannot be realized in experiments; we eliminate the AEs by lowering the neutral beam power. At $t=3.0 \mathrm{~s}$, the electron temperature profiles for the two shots are very close to each other, but the core electron density is $40 \%$ higher in pulse $94777\left(n_{\mathrm{e}}(0)=2.7 \times 10^{13} \mathrm{~cm}^{-3}\right)$ than in pulse $94771\left(n_{\mathrm{e}}(0)=1.6 \times 10^{13} \mathrm{~cm}^{-3}\right)$. This is partly due to the higher fuelling rate associated with the higher beam power, and partly due to the excellent particle confinement property of the transport barrier. Profiles of $q(\rho)$, the fast ion pressure, and various components of the plasma current density are also different. These results are shown in figure 8 . While $q(a)$ is fixed by the plasma current, which is the same for the two pulses, the $q$ profiles are different in the plasma core as shown in figure $8(b)$. It has been observed in TFTR [11] that fast ion ejection by the AEs happens in short bursts; one can infer from the neutron emission data [21] that the timeaveraged energetic ion pressure gradient is clamped at the critical value corresponding to the instability threshold. The same result emerges from TRANSP modelling depicted in figure $8(c)$. Despite the difference in the beam power, the modelled beam ion pressure profiles in the core are almost the same for the two pulses; the additional beam power in pulse 94777 is redistributed to the outer region of the plasma $(\rho=0.4-0.8)$ by the AEs as shown in figure 8(c). This kind of behaviour is consistent with the observations in the beam-driven TAE experiments in TFTR [21]. It gives rise to a higher beam-driven current in the same region as depicted in figure $8(d)$. Figure $8(e)$ shows that the bootstrap current is significantly higher in pulse 94777 just inside $\rho=0.4$ which is the ITB location. The bootstrap current is a significant part of the non-inductive current, but it is the total current density profile that determines the $q$-profile. The total core current density in pulse 94777 (with AEs) is significantly lower (figure $8(f)$ ) which corresponds to a significantly higher $q(0)$. The beam deposition profile from the charge exchange process peaks at $\rho=0$ in this low-density plasma, but the
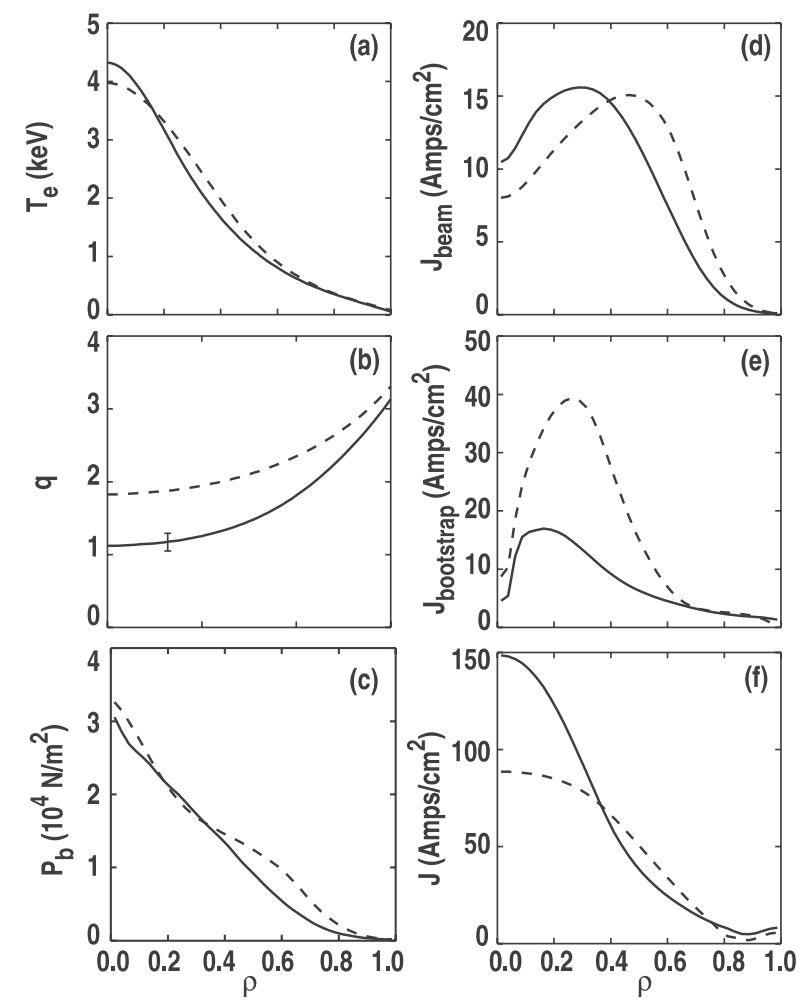

Figure 8. Comparison of two similar shots at $t=3 \mathrm{~s}$ with (shot $94777 ;-$ ) and without (shot $94771 ;-\ldots$ - Alfvén modes: Measured ( $a$ ) electron temperature profile, and $(b) q$-profile in the plasma core. Modelling results from TRANSP: $(c)$ beam ion pressure profile, $(d)$ beam-driven current density profile,

$(e)$ bootstrap current density profile and $(f)$ total current density profile.

AEs provide an off-axis current drive mechanism that tends to balance the inward diffusion of the inductive current and maintains $q(0)$ significantly above 1 until the end of the $5 \mathrm{~s}$ beam pulse. The electron temperature profile in figure $8(a)$ is measured by ECE calibrated by Thomson scattering; the experimental uncertainty is within $10 \%$. The $q$-profile in figure $8(b)$ is measured by MSE and the error bar is \pm 0.1 . All the other results come from TRANSP modelling which is tightly constrained by experimental measurements. The TRANSP code has matured after decades of development and this analysis technique is generally accepted in our field, but the uncertainty in the result is usually not easy to quantify. Nevertheless, it provides a self-consistent picture in agreement with the mechanism depicted in figure 2 .

\section{Reactor relevance}

The fact that $q(0)$ stays significantly above 1 , and the ITB in the plasma can last as long as the $5 \mathrm{~s}$ neutral beam pulse is very encouraging. It is important to find out whether this mechanism can be deployed in a burning plasma to form a steady-state NCS configuration with good confinement.

In high-performance plasmas from the TFTR tokamak where the pressure profile is strongly peaked [4], the major contribution to the radial electric field usually comes from the plasma pressure gradient. The gradient of the radial electric field $\left(\sim E_{\mathrm{r}} / a\right)$ gives rise to the shear in the poloidal flow that 
stabilizes the ITG turbulence. In the DIII-D tokamak, the major contribution to the radial electric field usually comes from the plasma toroidal rotation because all the beams have the same co-direction, and the shear of the rotation velocity gives rise to the shear in poloidal flow that stabilizes the ITG turbulence. These stabilization mechanisms are effective in these tokamaks because their minor radii are not too large. In a reactor size tokamak like ITER [22,23], the minor radius is 3 to 4 times bigger; $33 \mathrm{MW}$ of $1 \mathrm{MeV}$ neutral beam power is not high enough to drive a strong rotation and the gradient of $E_{\mathrm{r}}$ is probably not strong enough to stabilize the ITG turbulence. After ignition, energetic alpha particles are produced in the plasma core with almost isotropic velocity distribution. Contrary to a common misconception, only the co-moving energetic $\alpha$ particles are ejected by the Alfvén modes. This is because the energetic $\alpha$ particle pressure profile is centrally peaked, only those Alfvén modes propagating parallel to the plasma current can be excited $[13,24]$. A fraction of the co-moving energetic $\alpha$ particles can resonate with the Alfvén modes and get ejected to a larger radial location, leaving behind more counter-moving $\alpha$ particles in the core. Therefore, the basic mechanism to reduce the central magnetic shear remains unchanged. This is supported by the experimental results from TFTR $[11,21]$ which, unlike DIII-D, had balanced neutral beam injection.

Quantitative self-consistent model for AE-induced fast ion transport has not yet been developed. Nonlinear effects are expected to play an important role [25-27]. Unlike $\alpha$-driven TAE experiments in the past $[28,29]$, the $\alpha$ particle orbit is small compared to the minor radius in a reactor. Many overlapping Alfvén modes would be needed to sweep the energetic $\alpha$ particles from the reactor core to the outer region over a distance $\Delta r \sim a / 2$. An external antenna [30] may be needed to excite some of these modes. At this juncture, let us assume the most ideal situation that we can place the fast $\alpha$ particles anywhere we wish, and check if there is enough power to sustain the current density profile for NCS configuration. Analytic formulae based on bounce-averaged Fokker-Planck calculations are used to account for the electron screening current, trapped particle effects, energy diffusion and chargeexchange effects [31]. Using the DIII-D parameters, we find it quite easy to form NCS plasmas with co-beam [deuterium] injection provided ideal placement of the beam ions. In real DIII-D experiments, a significant fraction of fast ions ejected by the Alfvén modes leave the plasma and hit the vacuum vessel because of their large orbits [18]. This is probably why flat central shear instead of NCS plasmas were produced. Nevertheless, it serves our purpose because a quasi steady-state ITB is sustained without ballooning modes. In burning reactor plasmas, the energetic $\alpha$ particles are born with an isotropic velocity distribution, and they are doubly charged. Their current drive efficiency is significantly lower than deuterium co-beam injection because the fraction of co-moving particles is smaller and the electron screening effect [31] is larger for the doubly charged $\alpha$ particles. Crude estimates based on the current drive efficiency formula in [31] reveal that the $\alpha$ particle density in ITER may not be enough to form NCS plasmas. This depends on the initial current density profile, of course. If the initial central magnetic shear is weak, it is easy to make it negative. If the initial central shear is strongly positive,
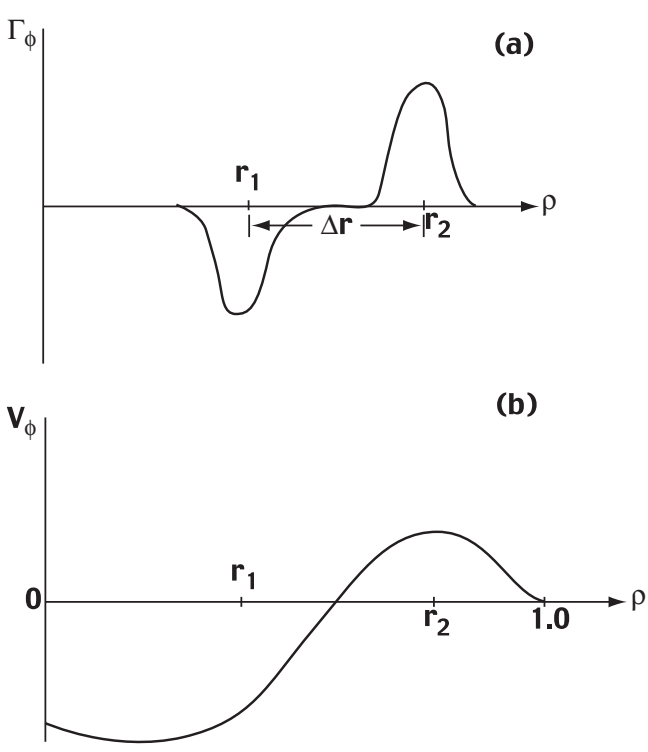

Figure 9. Schematic illustration of flow shear generation: $(a)$ torque on plasma due to co-moving ions ejected from $r_{1}$ to $r_{2}$. (b) Toroidal rotation velocity expected from the torque. The plasma edge at $\rho=1$ is assumed stationary.

it would require significantly more non-inductive current to reverse the shear. The chance of achieving NCS configuration improves if we change the machine parameters to optimize such a scheme. Even if the fast $\alpha$ particles can only sustain part of the non-inductive current needed for the NCS configuration, it still can reduce the external input power needed for current drive. It offers a new technique to control the current density profile through burn control or some enhanced fast $\alpha$ particle transport processes.

Although most of the ITB experiments were carried out in NCS configuration, NCS is not absolutely necessary for the existence of ITB [9]. One can avoid ballooning modes even in very steep pressure gradients when the shear is positive but low enough. In NCS plasmas, Alfvén cascade [32-34] at $q_{\min }$ can be a dominant mechanism for redistribution of the fast ions near $q_{\mathrm{min}}$.

An NCS configuration is not sufficient to form an ITB; a strong poloidal flow shear may also be needed. When a significant fraction of the co-moving energetic $\alpha$ particles are ejected from the core to the outer region of the plasma by the Alfvén modes, the outer region will rotate in the co-direction. There will be more counter-moving fast $\alpha$ particles left in the core, making the core plasma rotate in the counter direction as shown in figure 9 . The shear in toroidal rotation will raise the poloidal flow shear and the gradient in $E_{\mathrm{r}} . \alpha$ particles are less efficient than deuterium beams for current drive, but they are more efficient to drive plasma rotation because of the larger momentum per particle. This is true even when they have the same kinetic energy due to the larger $\alpha$ particle mass $\left[p=(2 m E)^{1 / 2}\right]$.

We calculate the upper bound of the $E_{\mathrm{r}}$ gradient based on the work of Rosenbluth and Hinton [35]. To get a quantitative estimate of the momentum transfer to the bulk plasma when a fraction of the fusion $\alpha$ particles are moved to the outer plasma region, we use an $\alpha$ distribution function calculated for a typical ITER steady-state plasma [36]. All co-passing $\alpha$ particles in 


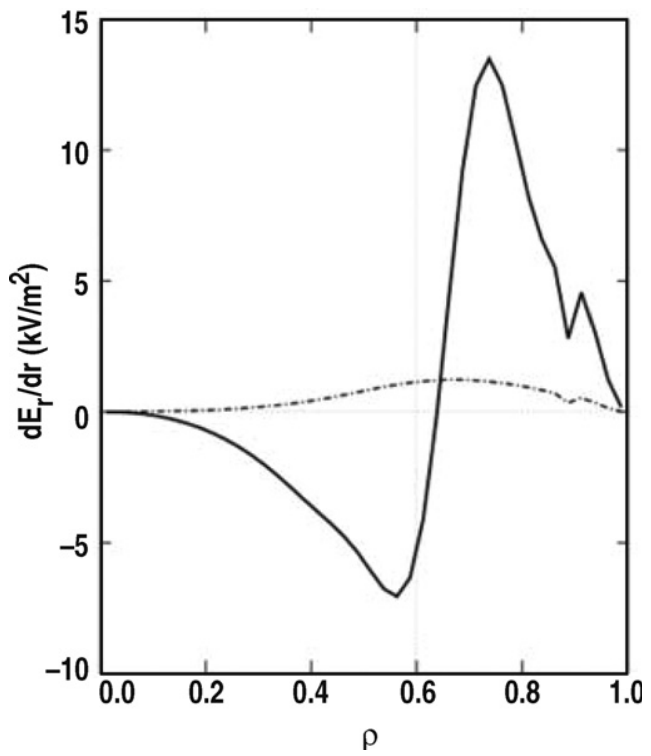

Figure 10. Gradient of the radial electric field due to the unperturbed (-. - ) and perturbed ( - ) fusion $\alpha$ particles in a typical steady-state ITER plasma. The ad hoc perturbation of the $\alpha$ distribution function moved all co-moving fusion $\alpha$ particles with $r / a<0.6$ to $r / a=0.6$.

a given energy range and with $r<r_{\text {move }}$ are moved into a radial shell of width $\Delta r / a=0.1$ at $r / a=r_{\text {move }}$. Here, $r$ is an effective minor radius variable derived from the plasma volume. In steady-state, the $\alpha$ particles apply a collisional torque to the plasma electrons which balances the viscous drag of the plasma [35],

$\int m_{\alpha} v_{\|} v_{\alpha \mathrm{e}} f_{\alpha}(W, \lambda, r) \mathrm{d} E \mathrm{~d} \lambda=m_{\mathrm{i}} n_{\mathrm{i}} \frac{1}{r} \frac{\partial}{\partial r}\left(r \chi_{\mathrm{m}} \frac{\partial v_{\phi}}{\partial r}\right)$.

Here $f_{\alpha}$ is the modified distribution function, which is a function of energy $W$, pitch $\lambda$ and position $r, m_{\alpha} v_{\|}$is the parallel momentum of an $\alpha$ particle, $v_{\alpha \mathrm{e}}$ is the slowing down rate of the $\alpha$ particles on electrons (obtained from the TRANSP simulation) and $\chi_{\mathrm{m}}$ is momentum diffusivity coefficient (assumed to be equal to the TRANSP ion thermal diffusivity [37]. We evaluate equation (1) to obtain the toroidal flow shear $\partial v_{\phi} / \partial r$, which is related to the shear in the radial electric field by the formula $\partial E_{\mathrm{r}} / \partial r=B_{\theta} \partial v_{\phi} / \partial r$. Results from this calculation, when all co-passing $\alpha$ particles with effective minor radius less than $r / a=0.6$ are moved to $r / a=0.6$, are shown in figure 10 .

A barrier is formed when the shearing rate $\gamma_{E \times B}$ exceeds the maximum linear growth rate $\gamma_{\mathrm{L}}$ of the ITG turbulence [6-8]. Using the plasma parameters in the ITER final design report and assuming the maximum linear growth rate $\gamma_{\mathrm{L}}$ to be $1 \%$ of the ion diamagnetic frequency with $k_{\theta} \rho_{\mathrm{i}}=1$, the obtained shearing rate becomes comparable to $\gamma_{\mathrm{L}}$. One can reduce $\Delta r$ to enhance the velocity shear if necessary. Based on these crude estimates, ITB formation with this scheme may be possible in ITER. Success is more likely in a reactor with higher energetic $\alpha$ density. After the barrier is formed, the criterion $\gamma_{E \times B}>\gamma_{\mathrm{L}}$ may be violated, but the barrier is expected to remain intact. Such hysteresis phenomenon has been observed in previous experiments [38]. The above calculation shows that the criterion for ITB formation is only marginally satisfied in ITER. However, when complete stabilization of ITG turbulence is not achieved, partial stabilization is still beneficial to plasma confinement, as pointed out in [38].

\section{Discussions}

Alfvén instabilities are driven by the free expansion energy of the energetic ions. In the case of our interest, the free expansion energy is only a small fraction $(\sim 1 \%)$ of the kinetic energy which is not tapped by the AEs. From the power balance point of view, it is apparent that significant bulk plasma heating by these AEs is not possible unless one can tap the kinetic energy of the energetic ions via instabilities driven by the gradient in velocity space $[39,40]$. $\alpha$ channelling involves phase space engineering, it modifies the $\alpha$ distribution function to accomplish some useful function in addition to electron heating. If an ITB is formed this way, its accomplishment is comparable to complete channelling of the $\alpha$ energy to ion heating. Therefore, this process is called $\alpha$ channelling in spite of the fact that only a small fraction of the $\alpha$ energy is involved [41]. When a significant amount of $\alpha$ particles are ejected quickly from the core to the edge, a bump-ontail $\alpha$ distribution is formed at the edge, and some velocity space instabilities may appear spontaneously to absorb the kinetic energy of the energetic $\alpha$ particles and restore the local $\alpha$ distribution towards a stable configuration. It would be desirable to exert some control of this process to direct the energy and momentum of the ejected $\alpha$ particles for the best effects. One can externally launch waves with appropriate frequency and parallel wavelength into the plasma and let them be amplified by the bump-on-tail $\alpha$ distribution. It would be highly desirable for the amplified waves to propagate inward, absorbed by the bulk ions to enhance DT fusion reactivity and at the same time drive additional shear flow to strengthen the barrier [42]. More work is needed to identify such a mode.

Lower hybrid current drive is planned for current profile control in ITER. One can launch unidirectional lower hybrid waves to be absorbed by the energetic $\alpha$ particles [43] for additional flow shear generation. However, the momentum content of a lower hybrid wave is very low when $n_{\|}$is comparable to 1 [44], which is needed to avoid electron Landau damping at the edge. Compressional Alfvén wave has much higher ratio of momentum flux to power flux. For $50 \mathrm{MW}$ of unidirectional ICRF fast wave power in ITER, the toroidal momentum in the wave is roughly $20 \%$ of the co-going alpha particle momentum. With proper choice of frequency and magnetic field, fast wave absorption can be highly localized and precisely controlled. It can be combined with the $1 \mathrm{MeV}$ neutral beams to fine tune the toroidal rotation velocity profile. This two-wave $\alpha$ channelling process is schematically depicted in figure 11. The difference between this and the two-wave scheme described in [2] is that the emphasis here is placed on ITB formation more than on ion heating. IBW tends to heat the ions in the bulk rather than in the tail of the distribution, therefore the reactivity enhancement is not as large. Previous IBW experiments have shown that the benefit from ITB formation can exceed that from direct ion heating [45]. It is important to note that when the energy and momentum of the ejected $\alpha$ particles are absorbed by the externally launched waves, the $E_{\mathrm{r}}$ shear will be different from what is shown in 

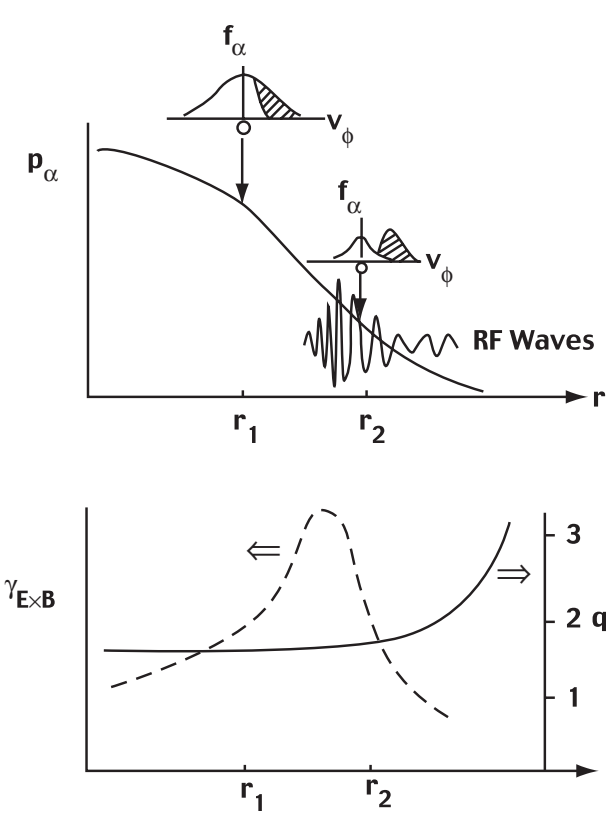

Figure 11. Schematic of a two-wave $\alpha$ channelling scheme similar to that described in [2]. The top panel shows the $\alpha$ pressure profile, and the bottom panel shows the shearing rate and the $q$-profile.

figure 10, which assumes that the energy and momentum of the ejected $\alpha$ particles are dissipated by electron drag. The main advantage of this $\alpha$ channelling process comes from the confinement improvement due to the ITB; enhancement of fusion reactivity becomes a secondary effect after higher $\beta$ is achieved. Since redistribution of energetic $\alpha$ particles is a spontaneous process in an ignited tokamak, it offers the possibility of an ITB as a natural steady-state of the burning plasma. We can envision the following scenario: after we start up the tokamak reactor, tritium fuel is introduced and centrally peaked $\alpha$ particle population builds up beyond the $\mathrm{AE}$ instability threshold. Co-going $\alpha$ particles are ejected from the core to the edge, set up an ITB which stays there throughout the fusion burn. This simplified picture is optimistic and forward looking, of course. It may happen in some operating parameter regime with an appropriate startup scenario. The details remain to be worked out. An extensive modelling study is needed to find out how this scheme can be effectively deployed in a fusion reactor.

\section{Summary}

Alfvén instabilities are usually considered undesirable because they enhance energetic ion loss, raise the possibility of first wall damage and reduce the $\alpha$ heating efficiency in burning plasmas. Here we demonstrate the possibility of using these instabilities as a means to produce NCS plasmas and raise the poloidal flow shear; these are essential ingredients to form ITB for better plasma confinement. The co-moving energetic $\alpha$ particles are swept from the core to the periphery. This selective transport process creates an off-axis current drive mechanism which can reduce or reverse the central magnetic shear and, at the same time, induce the plasma shear flow for ITB formation. This is a new scheme for steady-state ITB, never considered previously [46]. The DIII-D data presented here demonstrate that such a process can, indeed, occur in tokamaks. TRANSP modelling results indicate that the beamdriven current in the plasma core is reduced by $25 \%$ when AEs are excited in DIII-D, and the percentage changes in the total current density and the safety factor in the plasma core are even higher (see figure 8). However, further improvement based on the two-wave $\alpha$ channelling process remains to be a challenge for future experiments.

Finally, it should be pointed out that fishbone activities can trigger ITBs and sustain the local $q$-profile as observed in ASDEX Upgrade [47]. The scheme discussed in this paper relies on the redistribution of energetic ions in passing orbits whose effect on the non-inductive current is well understood. This is the process for neutral beam current drive in tokamaks, where experimental results have been repeatedly checked against Fokker-Planck calculations in the past and found good agreement. Fishbone oscillations are driven by trapped energetic ions. The non-inductive current arising from the redistribution of trapped energetic ions are equally interesting but less understood. In addition to fishbones, energetic trapped ions can also excite AEs [13], which in turn modifies the energetic ion pressure profile. If one fully understands the change in non-inductive current associated with this process, one can modify or control the $q$-profile by the AEs excited during ICRF heating [13]. This would be an important topic for the future.

\section{Acknowledgment}

This work is supported by the US Department of Energy under contracts DE-AC02-76CH03073 and DE-AC03-99ER54463.

\section{References}

[1] Fisch N.J. and Rax J.M. 1992 Phys. Rev. Lett. 69612

[2] Fisch N.J. and Herrmann M.C. 1995 Nucl. Fusion 351753

[3] Fisch N.J. and Herrmann M.C. 1994 Nucl. Fusion 341541

[4] Leviton F.M. et al 1995 Phys. Rev. Lett. 754417

[5] Strait E.J. et al 1995 Phys. Rev. Lett. 754421

[6] Walse R.E. et al 1994 Phys. Plasmas 12229

[7] Hahm T.S. and Burrell K.H. 1995 Plasma Phys. 21648

[8] Mazzucato E. et al 1996 Phys. Rev. Lett. 773145

[9] Gormezano C. et al 1998 Phys. Rev. Lett. 805544

[10] Greene J.M. and Chance M.S. 1981 Nucl. Fusion 21453

[11] Wong K.L. et al 1991 Phys. Rev. Lett. 661874

[12] Heidbrink W.W. et al 1991 Nucl. Fusion 311635

[13] Wong K.L. 1999 Plasma Phys. Control. Fusion 41 R1-R56

[14] Heidbrink W.W. 1995 Plasma Phys. Control. Fusion 37937

[15] Liu Chen 1994 Phys. Plasmas 11519

[16] Heidbrink W.W. et al 1999 Phys. Plasmas 61147

[17] Gorelenkov N.N. and Heidbrink W.W. 2002 Nucl. Fusion 42150

[18] Duong H.H. et al 1993 Nucl. Fusion 33749

[19] Hawryluk R.J. 1980 Physics of Plasmas Close to Thermonuclear Conditions vol 1, ed B. Coppi (Brussels: ECE) p 19

[20] St John H. et al 1993 Proc. 20th EPS Conf. on Controlled Fusion and Plasma Physics (Lisboa, 1993) (part I) p 99

[21] Wong K.L. et al 1992 Phys. Fluids B 42122

[22] Campbell D.J. 2001 Phys. Plasmas 82041

[23] Aymar R. 2001 Nucl. Fusion 411301

[24] Candy J. et al 1997 Phys. Plasmas 42597

[25] Berk H.L. et al 1996 Phys. Plasmas 31827

[26] Todo Y. et al 2003 Phys. Plasmas 102888

[27] Zonca F. et al 2002 Phys. Plasmas 94939 
[28] Wong K.L. et al 1996 Phys. Rev. Lett. 762286

[29] Nazikian R. et al 1997 Phys. Rev. Lett. 782976

[30] Fasoli A. et al 1995 Phys. Rev. Lett. 75645

[31] Okano K. 1990 Nucl. Fusion 30423

[32] Berk H.L. et al 2001 Phys. Rev. Lett. 87185002

[33] Breizman B.N. et al 2003 Phys. Plasmas 103649

[34] Nazikian R. et al 2003 Phys. Rev. Lett. 91125003

[35] Rosenbluth M.N. and Hinton F.L. 1996 Nucl. Fusion 3655

[36] Budny R.V. 2002 Nucl. Fusion 421383

[37] Budny R.V. et al 1995 Nucl. Fusion 351497
[38] Synakowski E.J. et al 1997 Phys. Rev. Lett. 782972

[39] Gates D.A. et al 2000 Phys. Rev. Lett. 87205003

[40] Gorelenkov N.N. et al 2003 Nucl. Fusion 43228

[41] Fisch N.J. 2004 Princeton University, private communication

[42] LeBlanc B.P. et al 1999 Phys. Rev. Lett. 82331

[43] Wong K.L. and Ono M. 1984 Nucl. Fusion 24615

[44] Ohkawa T. 1970 Nucl. Fusion 10185

[45] LeBlanc B. et al 1995 Phys. Plasmas 2741

[46] Connor J.W. et al 2004 Nucl. Fusion 44 R1

[47] Guenter S. et al 2001 Nucl. Fusion 411283 